\title{
ANALYSIS OF HYDROGEN BUBBLE FLOW BETWEEN NANORODS USING LATTICE BOLTZMANN METHOD
}

\author{
HEDVIG PARADIS ${ }^{1}$, COSTAS GRIGOROPOULOS $^{2}$ \& BENGT SUNDÉN ${ }^{1}$ \\ ${ }^{1}$ Department of Energy Sciences, Faculty of Engineering, Lund University, Box 118, 22100 Lund, Sweden. \\ ${ }^{2}$ Department of Mechanical Engineering, University of California, Berkeley, CA 94720, USA.
}

\begin{abstract}
Lattice Boltzmann method (LBM) is used to model the hydrogen production by splitting water by incident sunlight over Si nanorods. The purpose of this study is to investigate the transport and the formation of the hydrogen bubbles by electrochemical reactions with a $2 \mathrm{D}$ and a $3 \mathrm{D}$ numerical model using LBM. An ordered array of nanorods is created where each rod is $10 \mu \mathrm{m}$ in high and $10 \mathrm{~nm}$ in diameter. The numerical models are simulated using MATLAB and parallel computing with the program Palabos. A reaction-advection-diffusion transport for two components is analyzed with electrochemical reactions. This process is further coupled with the momentum transport. The effect of different bond numbers and contact angels on the simulation results are analyzed.

It has here been shown that LBM can be used to evaluate the transport processes at microscale and it is possible to include the effect of electrochemical reactions on the transport processes. An increased Bond number increases the bubble flow through the nanorod domain. A decreased contact angle facilitates the disconnection of the bubble to the nanorod at the top surface. The collection of the hydrogen bubbles at the top surface of the nanorods will be facilitated by an easy disconnection of the bubbles.

Keywords: LBM, bubble flow, microscale, mass diffusion, electrochemical reactions, Bond number, contact angle.
\end{abstract}

\section{INTRODUCTION}

Hydrogen is known to be a very efficient and useful fuel. But hydrogen needs to be developed from another source as it does not exist freely in the accessible atmosphere. Common ways to obtain hydrogen is through electrolysis using natural gas or hydrolysis using photocatalyst materials [1]. Hydrolysis can be carried out by photo-electrochemical systems, which produce hydrogen from water using sunlight and photo-electrochemical materials. The photo-electrochemical materials are specialized semiconductors that absorb sunlight and use the light energy to separate water molecules into hydrogen and oxygen. Current research focuses on developing a prominent design for a nanostructure hydrolysis device [1,2].

The device of interest in this study is designed with a membrane that separates the anode and the cathode and all three parts are embedded in $\mathrm{H}_{2} \mathrm{O}$. An illustration of the device is presented in Fig. 1. The semiconductor device consists of arrays of $p-n$ junction nanorods, which enable light absorption and carrier extraction. Each nanorod in the cell is long in the direction of incident light (z-direction), allowing for optimal light absorption, but thin in the other directions [2]. By attaching individual catalysts for water reduction and oxidation to the membrane, the process of splitting water is divided into separate reduction and oxidation reactions [3]. The catalysts are attached on opposite sides of the membrane. Each catalyst can, therefore, be optimized independently for a single reaction. Inexpensive ordered $\mathrm{Si}$ wire arrays can be grown on a single crystal wafer and transferred into a flexible polymer matrix [2].

To make a functional water splitting system, the device is built up on two semiconductors on opposite sides of a membrane. Heterogeneous electrochemical catalysts from earth abundant or non-noble metals can be used to convert the incoming light to $\mathrm{H}_{2}(\mathrm{~g})$ in an aqueous solution on p-type Si nanorod arrays. Homogeneous electrochemical catalysts can be made 


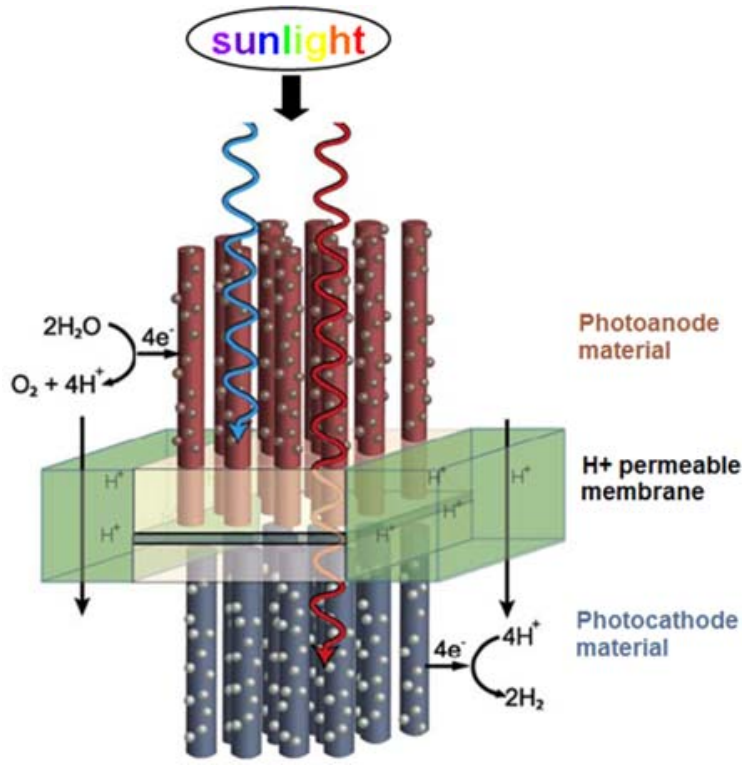

Figure 1: Schematic figure of the semiconductor device [3].

to operate near the thermodynamic potential for hydrogen evolution $[2,3]$. The bubble formation and generation result in a complex set of optical and electrochemical phenomena at the semiconductor-catalyst-electrolyte interfaces. Semiconductor photo-electrodes for water splitting are most efficient with surface features such as high-surface-area morphologies or heterogeneous catalysts $[3,4]$.

The goal of this study has been to develop a numerical model, using LBM, of a watersplitting photo-conversion system that can produce hydrogen fuel from sunlight and water. The focus here is on the formation and movement of the bubbles between the rods toward the top part of the geometry. In the actual system, the bubbles are created when the sunlight strikes an active part of the nanorod by enabling the electrochemical reaction. When the bubble is created, it is important to provide physical conditions and design for the bubbles to rise between the rods without getting re-attached to the rods or re-react at an active site. Therefore, the specific motivation for this study is to develop a numerical model for feasible physical conditions to collect hydrogen bubbles. Note that the sunlight is not taken into account in the numerical model. In the numerical model, the formation of the bubbles is handled by implementing a reaction rate at the bottom surface of the nanorods.

\section{GOVERNING EQUATIONS}

The species involved for the whole system are water, hydrogen and oxygen. The species reactive transport is initiated with a water $\left(\mathrm{H}_{2} \mathrm{O}\right)$ molecule, which reacts through the solid nanorods on the anode side by solar light. $\mathrm{H}_{2} \mathrm{O}$ forms positive hydrogen protons during this reduction reaction and releases an oxygen molecule. The electrons generated at the anode are conducted through the photocatalyst materials. The protons enter the cathode through the membrane and form hydrogen with the electrons by the interface reaction sites [3].

$$
2 \mathrm{H}_{2} \mathrm{O} \rightarrow \mathrm{O}_{2}+4 \mathrm{H}^{+}+4 e^{-}
$$




$$
4 H^{+}+4 e^{-} \rightarrow 2 H_{2}
$$

There are three main equations modeled in this study. First, the reaction-advectiondiffusion equation can be written as $[4,5]$ :

$$
\frac{\partial C_{i}}{\partial t}+(\boldsymbol{u} \cdot \nabla) C_{i}-D \nabla^{2} C_{i}=R_{r}
$$

where $C_{i}$ is the concentration of species $i, D$ is the diffusion coefficient, $R_{r}$ is the reaction rate for reaction with the specific species $i, \boldsymbol{u}$ is the velocity vector and $t$ is the time. Secondly, the Navier-Stokes equations for conservation of momentum and the conservation of mass are presented as $[4,5]$ :

$$
\begin{gathered}
\frac{\partial \boldsymbol{u}}{\partial t}+(\boldsymbol{u} \cdot \nabla) \boldsymbol{u}+\frac{\nabla p}{\rho}-v \nabla^{2} \boldsymbol{u}=\boldsymbol{F} \\
\nabla \cdot \boldsymbol{u}=0
\end{gathered}
$$

where $p$ is the pressure in the fluid, $v$ is the kinematic viscosity, $\mathbf{F}$ is the force term, $u$ is the velocity and $t$ is the time. The Bond number is used to characterize the shape of the bubble in the surrounding fluid. Bond number is here defined as [6]

$$
B o=\frac{\Delta \rho \cdot g \cdot L^{2}}{\gamma}
$$

where $\Delta \rho$ is the difference in density between the gas and the fluid, $g$ the gravitational acceleration, $L$ the characteristic length and $\gamma$ the surface tension. Bond number Bo is the ratio of body forces (here gravitational force) and the surface tension. It can be viewed as a dimensional number for the bubble [6].

The current density is connected to the concentration gradients via the source terms in the reaction-diffusion-advection equation. The electrochemical reactions are implemented by source terms $[7,8]$.

$$
\begin{aligned}
R_{\mathrm{H}_{2}} & =\frac{-i}{2 \cdot F} \\
R_{\mathrm{H}_{2} \mathrm{O}} & =\frac{i}{2 \cdot F}
\end{aligned}
$$

where $i$ is the current density and $F$ the Faraday constant. Here, a constant current density is assumed at the cathode-membrane interface and it is connected through the reaction rate terms in the reaction-diffusion-advection equation.

\section{LATTICE BOLTZMANN MODEL}

Lattice Boltzmann method (LBM) has shown the ability to handle complex multiphysical and multiphase problems as well as complex geometries. A common historical approach is to use interface models that are in certain cases likely to be incorrect if applied to processes on length scales similar to the thickness of the interface. Such cases include motion near some critical point, motion of a contact line between a liquid and vapor along a solid wall or spontaneous formation of bubbles.

LBM is based on the Boltzmann equation and is perceived as an alternative to the traditional computational fluid dynamics (CFD) based on the Navier-Stokes equation. LBM 
simulates transport phenomena by tracking movements of molecule ensembles and the evolution of the distribution functions [9]. LBM has shown promising simulation results of fluid flows and mass diffusion through complex geometries. Conventional CFD methods use fluid density, velocity and pressure as the primary variables, whereas the LBM uses a more fundamental approach with the so-called particle velocity distribution function (PDF) [10, 11]. In spite of LBM's simplicity, it has a disadvantage of taking up more memory than a traditional CFD approach. For this case, parallel computing offers the possibility to include several physical processes in the model with success.

LBM's main function, PDF, is defined as the number of particles of the same species traveling along a particular direction with a particular velocity. LBM is built up on lattice points that are given locations placed all over the regularized solution domain. The LBM is described by two different actions taking part at each lattice point (site); namely streaming and collision $[10,11]$. Streaming describes the movement of the particles of each species and collision describes interactions between the particles of the same or different species. Further, these actions are combined in the LB equation for species $i[4,7,10,11]$

$$
f_{a}^{i}\left(x+e_{a}^{i} \Delta t, t+\Delta t\right)=f_{a}^{i}(x, t)+\Omega_{a}^{i}(x, t)+F_{a}^{i}(x, t)
$$

where $f_{a}^{i}$ is the PDF, $e_{a}^{i}$ the discrete streaming velocity, $\Omega_{a}^{i}$ the collision term, $\Delta t$ the simulation time step and $F_{a}^{i}$ the source term at any spatial location $x$ and time $t$ along the direction $\alpha$. The collision term and the equilibrium function are specified as

$$
\begin{aligned}
\Omega_{a}^{i}(x, t) & =-\frac{f_{a}^{i}(x, t)-f_{a}^{i, e a}(x, t)}{\tau_{i}} \\
f_{a}^{i, e a} & =w_{a} \cdot C_{i}\left[1+3 \cdot e_{a} \cdot u_{i}\right] \\
u_{i} & =\frac{\sum_{i} \frac{1}{\tau_{i}} \cdot \sum_{a} f_{a}^{i} e_{a}}{\sum_{i} \frac{1}{\tau_{i}} C_{i}}
\end{aligned}
$$

Where $w_{a}$ is the weight factor due to the placement of the particles in the grid, $u_{i}$ is the specific velocity contribution and $\tau_{i}$ is the relaxation time for the specific species $i$. Here, the number of directions is 19 for the conservation of momentum and mass. The number of directions is 7 for the reaction-advection-diffusion equation. In this case, the source term includes the effect of the chemical reactions. The source term is shown below $[5,12,13]$ :

$$
F_{a}^{i}(x, t)=w_{a} \cdot \Delta t \cdot R_{r}
$$

where $R_{r}$ is the reaction rate in lattice units for the $r$ th reaction, $\Delta t$ and $\Delta x$ are the time step and length step in the simulation, respectively. These are defined in SI units, (s) seconds and (m) meters, respectively. The concentration $C$ is obtained directly in $\left(\mathrm{mol} / \mathrm{m}^{3}\right)$, which has been shown by several authors [11-15]. The concentration is obtained by

$$
C_{i}=\sum_{a=0}^{6} f_{a}^{i}(x, t)
$$

Streaming describes how the particles proceed. Collision can be between particles of the same species or particles of different species or with the wall [9]. 
Table 1: Analysis parameters.

\begin{tabular}{ll}
\hline Parameter & Value \\
\hline Cell length & $30 \mu \mathrm{m}$ \\
Rod length & $10 \mu \mathrm{m}$ \\
Rod diameter & $0.01 \mu \mathrm{m}$ \\
Diffusivity $\mathrm{D}_{\mathrm{H} 2 \mathrm{H} 2 \mathrm{O}}$ & $4 \mathrm{e}-5 \mathrm{~m}^{2} / \mathrm{s}$ \\
Viscosity of $\mathrm{H}_{2} \mathrm{O}$ & $1.5 \mathrm{e}-5 \mathrm{~m}^{2} / \mathrm{s}$ \\
Temperature & $273 \mathrm{~K}$ \\
Pressure & $1 \mathrm{~atm}$ \\
Average current density & $80 \mathrm{~A} / \mathrm{m}^{2}$ \\
Number of grid points & $200 \times 80 \times 80$ \\
\hline
\end{tabular}

Similarly, for the Navier-Stokes equations a PDF, named $g_{i}$, is applied as

$$
\begin{gathered}
g_{\alpha}^{i}(x+\Delta x, t+\Delta t)=g_{\alpha}^{i}(x, t)+\Omega_{\alpha}^{i}(x, t)+G_{\alpha}^{i}(x, t) \\
\rho=\sum_{\alpha=0}^{6} g_{\alpha} \\
g_{\alpha}^{i, e q}(x)=w_{\alpha} \cdot \rho_{i}\left[1+3 \frac{e_{\alpha} \cdot v_{i}}{c^{2}}+\frac{9}{2} \frac{\left(e_{\alpha} \cdot v_{i}\right)^{2}}{c^{4}}-\frac{3}{2} \frac{v_{i}^{2}}{c^{2}}\right] \\
v_{i}=\frac{1}{\rho} \cdot \sum_{\alpha=0}^{6} g_{\alpha}^{i} e_{\alpha}
\end{gathered}
$$

where $\rho$ is the density of the fluid and $v$ the fluid velocity. The force term $G$ is related to the gravitational acceleration $g$. For a detailed description of the free surface model using LBM, which is here implemented for the 3D model, the reader is referred to Thürey [16] and Pohl [17].

The geometry is created by placing ordered nanorods embedded in water. Note that only the cathode side is modeled in this study with focus on the bubble distribution between the nanorods. The membrane interface by the cathode is treated as an interface and for the 3D model the bubble formation takes place only as an interface condition.

In this study, LBM is applied to handle mass transport, more specifically the reactionadvection-diffusion equation. The most significant parameters concerning the cell structure and catalytic activity for the calculations are presented in Table 1.

\section{RESULTS AND EVALUATION OF LBM}

The modeling studies are performed in Palabos and part of it in MATLAB to compare the in-house codes to a commercial program. For the simulation of the bubble formation and distribution, a cluster provided by Lunarc at Lund University is used. The total number of time steps for the simulation for the bubble distribution is 80000 for all cases.

The geometry is built up by an ordered array of cylindrical rods. The electrochemical reactions are implemented as boundary conditions at the bottom surface and on the surface of the 


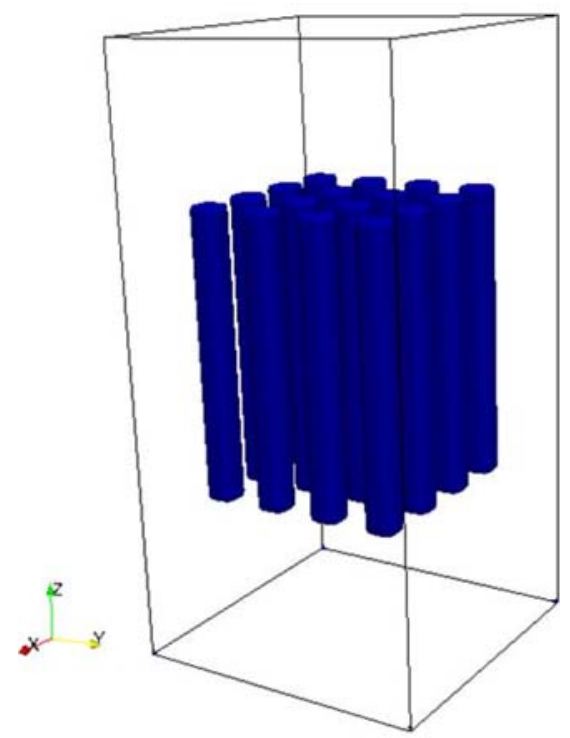

Figure 2: Schematic figure of the modeling domain in 3D.

rods for a small part close to the bottom surface. The bottom surface, under the rods, is where the membrane would be situated. A schematic figure of the modeling domain is presented in Fig. 2. Note that the membrane has not been included in this study. The modeling domain in all cases is $30 \mu \mathrm{m}$ in high in $z$-direction and $10 \mu \mathrm{m}$ in $x$ - and $y$-directions. The number of grid points is $200 \times 80 \times 80$. The number of grid points has been varied from $150 \times 60 \times 60$ to $300 \times 120 \times 120$, and no significant changes are found to have an impact on the simulation. The Reynolds number is $R e \leq 1$ for the modeled cases. The bubble distribution in 3D is shown in Figs 3-6 for different cases of Bo numbers and contact angles. The base case is Bo $=100$ with a contact angle of $90^{\circ}$. Note that the bubbles represent hydrogen and the surrounding fluid represents water but the fluid is not explicitly visualized. The bubble distribution is presented to illustrate the evolution of the bubbles and shape changes. The buoyancy moves the bubbles and there is no velocity parameter varied in the 3D model. However, for the 2D model a velocity parameter is applied. In Figs 6 and 7, the bubble formation is shown in 2D for $\mathrm{H}_{2}$ without and with electrochemical reactions, respectively.

In Fig. 3, the bubble distribution is visualized for $\mathrm{Bo}=1$ and a contact angle of $90^{\circ}$. In this case, the bubbles do not float up by the buoyancy effect because as the bubbles are formed, they become lumped together at the bottom of the nanorods throughout the simulation. However, the movement of the bubbles toward the top surface could have been improved by increasing the inlet velocity in the $z$-direction. The bubbles will remain more or less spherical during the simulation due to the low Re, which can be understood from a shape regime map presented in [8]. LBM can also handle the two-phase flow with the bubbles and the surrounding fluid in a domain with nanorods. However, in the 3D model only the surrounding fluid is explicitly simulated to minimize the computational cost. It is illustrated that LBM can easily handle the obstacles in the modeling domain by the bounce-back effect for the particles at the obstacle wall in the model. 


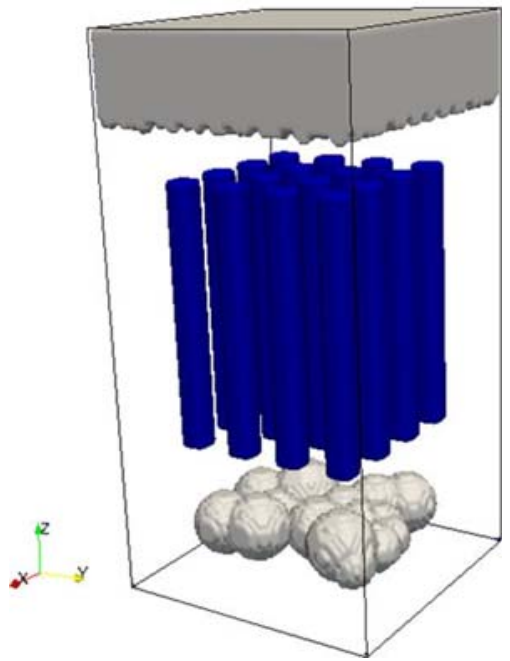

Figure 3: Bubble distribution for the 3D modeling domain for $\mathrm{Bo}=1$ and a contact angle of $90^{\circ}$ after 50000 time steps.

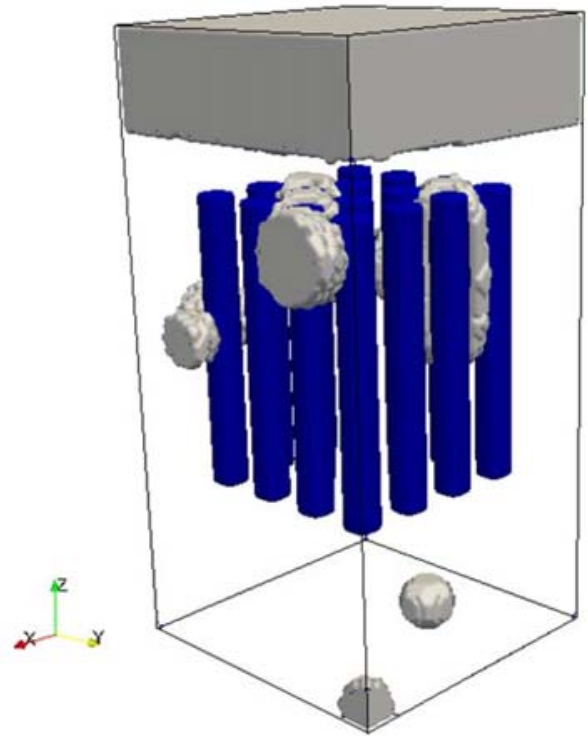

Figure 4: Bubble distribution for the 3D modeling domain for $\mathrm{Bo}=100$ and a contact angle of $90^{\circ}$ after 50000 time steps.

In Fig. 4, the bubble distribution is visualized for $\mathrm{Bo}=100$ and a contact angle of $90^{\circ}$. (The case for $\mathrm{Bo}=10$ and a contact angle $90^{\circ}$ is not presented here due to similarities to the case for $\mathrm{Bo}=100$ and a contact angle of $90^{\circ}$.) In this case, the bubbles do float up by the buoyancy effect but are lumped together during the passage between the nanorods during the simulation. 


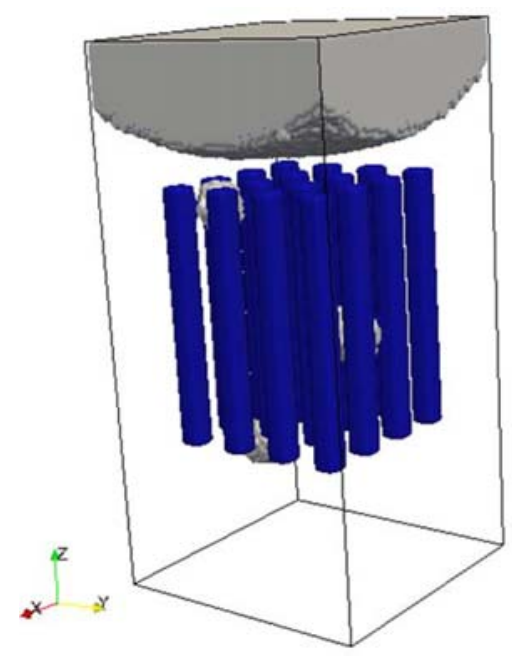

Figure 5: Bubble distribution for the 3D modeling domain for $\mathrm{Bo}=100$ and a contact angle of $60^{\circ}$ after 50000 time steps.

In this case as well, the movement of the bubbles toward the top surface could have been improved by increasing the inlet velocity in the $z$-direction. Still there is a need to overcome the problem that the bubbles tend to stick to the surface of the nanorods. When the bubbles stick to the nanorods, there is an increased risk of the bubbles re-reacting with active species and the bubbles will not be captured at the top surface of the nanorods.

In Fig. 5, the bubble distribution is visualized for $\mathrm{Bo}=100$ and a contact angle of $60^{\circ}$. Here the bubbles float up by the buoyancy effect and are not lumped together between the nanorods during the simulation. In this case, the movement of the bubbles is improved and there is not the same need of increasing the inlet velocity in the $z$-direction as for the other cases. The bubbles float up to the top domain where the bubbles are captured. This can be seen in Fig. 5 as an increased volume of the grey area at the top part of the modeling geometry.

The following section presents the bubble formation in 2D. The 2D images provide a more clear visualization of the concentration distribution of $\mathrm{H}_{2}$ and $\mathrm{H}_{2} \mathrm{O}$. The bubble movement toward the top surface has no direct difficulties in the $z$-direction but some of the bubbles are not completely developed and tend to stick to the surface of the nanorods. The velocity in the porous domain is low and it is fully laminar throughout the whole domain.

In Fig. 6, the concentration of the hydrogen bubble distribution is visualized for $\mathrm{Bo}=100$ and a contact angle of $90^{\circ}$ at the time step 50000 . For this case, the concentration difference is driven by advection and diffusion. The electrochemical reactions are handled as a boundary condition at the bottom surface, which means no electrochemical reaction takes place along the rods. Reynolds number is $R e \lesssim 1$. The high concentration indicates where the concentration of hydrogen is high. The low concentration spots in the passages are where the water is to be found in the mixture. It should not be confused with the darker blue color indicating the placement of the nanorods. Note that the 2D figure shows both passages and rods in Fig. 6 .

In Fig. 7, the concentration of the hydrogen bubble distribution is visualized for $\mathrm{Bo}=100$ and a contact angle of $90^{\circ}$ at time step 50000 . For this case, the concentration difference is driven by advection and diffusion as well as electrochemical reactions. The electrochemical 


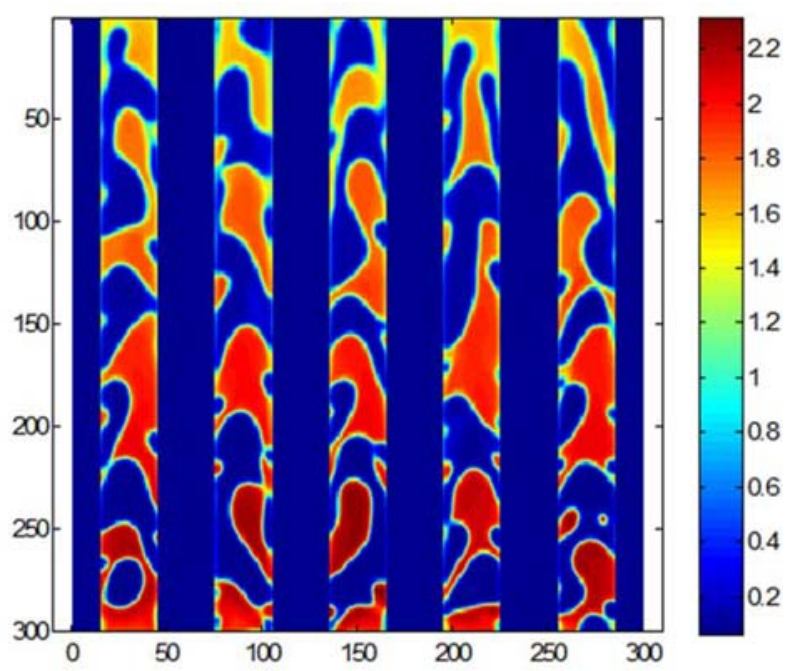

Figure 6: Concentration distribution $\left(\mathrm{mol} / \mathrm{m}^{3}\right)$ of $\mathrm{H}_{2}$ without electrochemical reactions for several rods and passages.

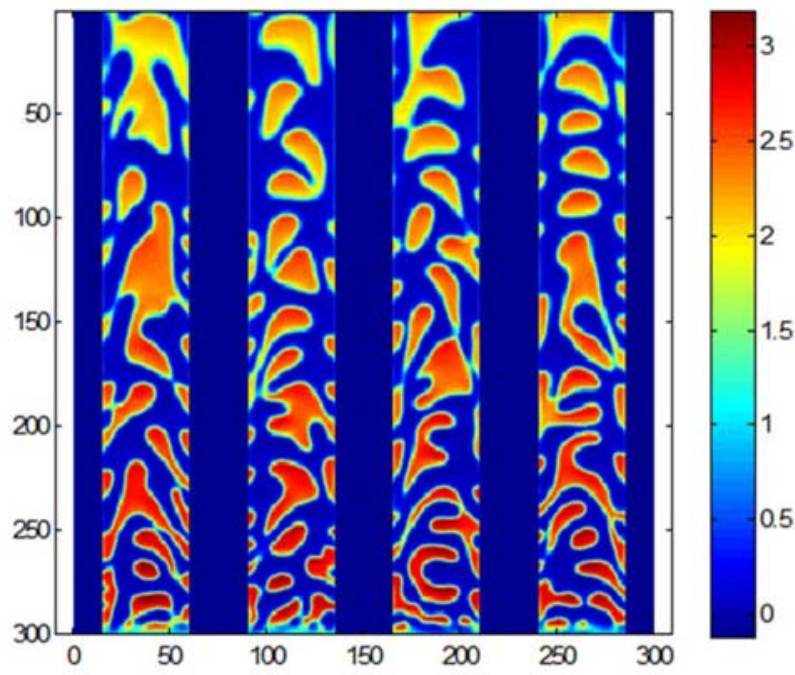

Figure 7: Concentration distribution $\left(\mathrm{mol} / \mathrm{m}^{3}\right)$ of $\mathrm{H}_{2}$ with electrochemical reactions for several rods and passages.

reactions take place at the bottom surface of the nanorods and the passages. The high concentration indicates where the concentration of hydrogen is high. The low concentration spots in the passages are where the water is to be found in the mixture. It should not be confused with the darker blue color indicating the placement of the nanorods. Note that the 2D image shows both passages and rods in Fig. 7. 
The main difference between the 3D and 2D plots are that only the bubble distribution is presented for the $3 \mathrm{D}$ case, but for the $2 \mathrm{D}$ case the concentration distribution of the species and the bubble distribution are presented. By comparing the bubble distribution in size and shape between the two models, it is shown that the 3D bubble distribution has more round (spherical) shaped bubbles. But for the 2D bubble distribution, the bubbles tend to have more randomly shaped configurations. One reason for this difference is that in the $2 \mathrm{D}$ model, the bubbles cannot move sideways between the rods. The bubbles can in this case only to follow the channel it is formed in. Also, an effect on the bubble distribution and bubble shape will occur when electrochemical reactions is included along the part of the rod. This phenomenon is not included in the 3D model.

\section{CONCLUSIONS}

In this study, momentum and mass transport with electrochemical reactions have been evaluated by LBM for water splitting over nanorods. The analysis level has been for microscale transport where transport phenomena with electrochemical reactions are recognized as truly important. In the 3D model, the focus is to evaluate LBM for a nanorod constructed geometry and for the bubble distribution of hydrogen. The effect on the bubble distribution by changing the Bond number and the contact angle is studied. In the 2D model, a reaction-diffusion process coupled with momentum transport is implemented. The electrochemical reactions are implemented as source terms using the reaction rate for the specific reaction.

It is concluded that the LBM can successfully be used to model this complex structure at smaller scales. As the Bond number is increased (up to 100), the bubble distribution is improved and the bubbles do not lump together until the upper part of the nanorods. The contact angle could be around $60^{\circ}$ to get fewer disturbances of lumped bubbles sticking to the surface of the nanorods. It could be easier to effectively distribute and move the bubbles through the nanorods with a slightly increased velocity in the main flow direction. However, this needs to be studied further by also including the full physical problem with specific parameters and equations. The concentration distribution for hydrogen including electrochemical reactions presents smaller and more frequent formations of hydrogen bubbles and the bubbles do not tend to stick to the walls as in the case where the effect of the electrochemical reactions were not included.

A detailed implementation with specific placement of the reaction with respect to the incoming sunlight would be valuable for future studies. Validation against experimental results, where images of the reactions at microscale can be viewed, would also be valuable.

\section{ACKNOWLEDGEMENTS}

The financial support from The Swedish Research Council (VR-621-2010-4581) and the European Research Council (ERC-226238-MMFCs) is gratefully acknowledged for financial support of this research work.

\section{NOMENCLATURE}

Bo Bond number

C concentration $\left(\mathrm{mol} / \mathrm{m}^{3}\right)$

$D$ diffusivity $\left(\mathrm{m}^{2} / \mathrm{s}\right)$

$d_{p} \quad$ particle diameter $(\mathrm{m})$

e $^{p} \quad$ base velocity in the lattice Boltzmann model (lu/ts)

$f \quad$ particle distribution function, momentum (-)

$F \quad$ Faradays constant $(96485 \mathrm{~A} \cdot \mathrm{s} / \mathrm{mol})$ 


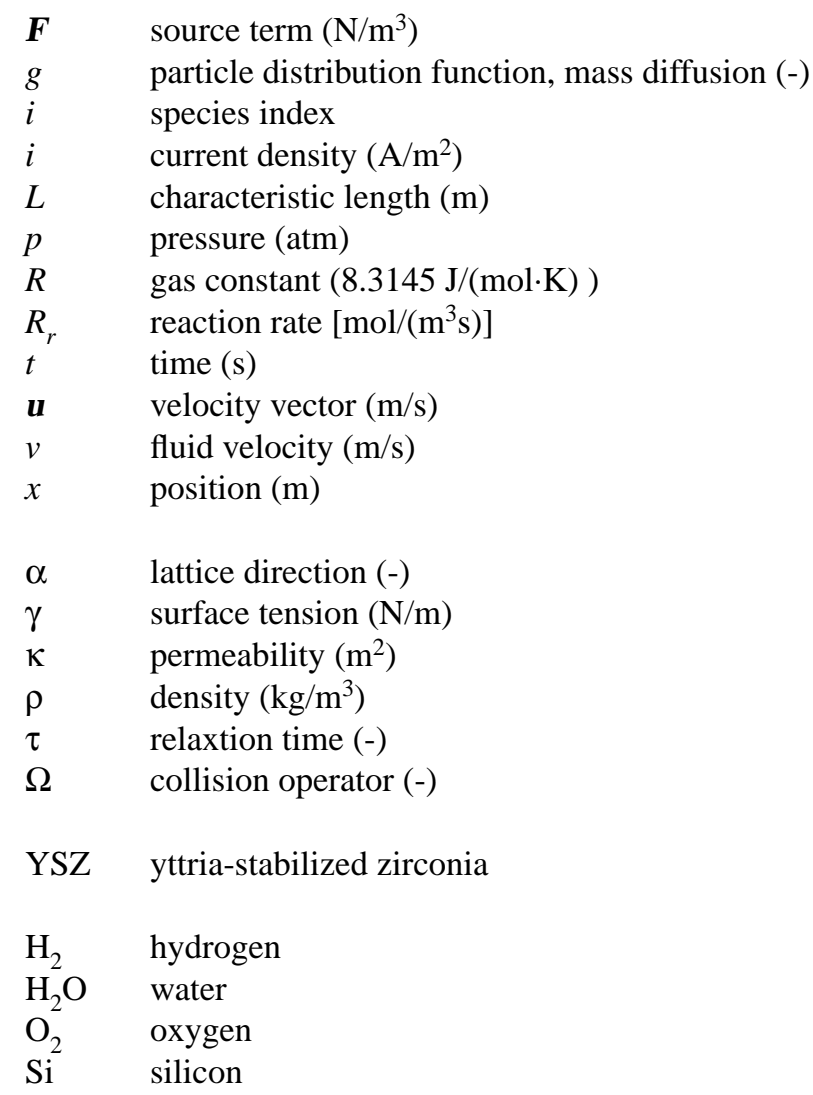

\section{REFERENCES}

[1] Momirlan, M. \& Veziroglu, T.N., Current status of hydrogen energy. Renewable and Sustainable Energy Reviews, 6, pp. 141-179, 2002. doi: http://dx.doi.org/10.1016/ S1364-0321(02)00004-7

[2] Walter, M.G., Warren, E.L., McKone, J.R., Boettcher, S.W., Mi, Q., Santori, E.A. \& Lewis, N.S., Solar water splitting cells. Chemical Review, 110, pp. 6446-6473, 2010. doi: http://dx.doi.org/10.1021/cr1002326

[3] Reyes Gil, K.R., Spurgeon, J.M. \& Lewis, N.S., Silicon and tungsten oxide nanostructures for water splitting. Proceedings of SPIE Solar Hydrogen and Nanotechnology IV, ed. F.E. Osterloth, Vol. 7404, 74080S-1-74080S-11, 2009. doi: http://dx.doi. org/10.1117/12.825545

[4] Dawson, S.P., Chen, S. \& Doolen, G.D., Lattice Boltzmann computations for reactiondiffusion equations. Journal of Chemical Physics, 98, pp. 1514-1523, 1993. doi: http:// dx.doi.org/10.1063/1.464316

[5] Xu, Y.-S., Zhong, Y.-J. \& Huang, G.-X., Lattice Boltzmann method for diffusionreaction-transport processes in heterogeneous porous media. Chinese Physics Letters, 21(7), 2004.

[6] Clift, R., Grace, J.R. \& Weber, M., Bubbles, Drops and Particles, Academic Press: New York, USA, 1978.

[7] Haussener, S., Xiang, C., Spurgeon, J.M., Ardo, S., Lewis, N.S. \& Weber, A.Z., Modeling, simulation, and design criteria for photoelectrochemical water-splitting systems. En- 
ergy \& Environmental Science, 5, pp. 9922-9935, 2012. doi: http://dx.doi.org/10.1039/ c2ee23187e

[8] Leenheer, A.J. \& Atwater, H.A., Water-splitting photoelectrolysis reaction rate via microscopic imaging of evolved oxygen bubbles. Journal of Electrochemical Society, 157, pp. B1290-B1294, 2010. doi: http://dx.doi.org/10.1149/1.3462997

[9] Joshi, A.S., Peracchio, A.A., Grew, K.N. \& Chiu, W.K.S., Lattice Boltzmann method for multi-component, non-continuum mass diffusion. Journal of Physics D: Applied Physics, 40, pp. 7593-7600, 2007. doi: http://dx.doi.org/10.1088/0022-3727/40/23/053

[10] Sukop, M.C. \& Thorne, D.J. Jr., Lattice Boltzmann Modeling - An Introduction for Geoscientists and Engineers, Springer: New York, USA, 2007.

[11] Latt, J., Hydrodynamic limit of the lattice Boltzmann equations. PhD Thesis, University of Geneva, 2007.

[12] Chen, S., Dawson, S.P., Doolen, G.D., Janecky, D.R. \& Lawniczak, A., Lattice methods and their applications to reacting systems. Computers and Chemical Engineering, 19, pp. 617-646, 1995. doi: http://dx.doi.org/10.1016/0098-1354(94)00072-7

[13] Kang, Q.J., Lichtner, P.C. \& Janecky,D.R., Lattice Boltzmann method for reacting flows in porous media. Advances in Applied Mathematics and Mechanics, 5, pp. 545$563,2010$.

[14] Shan, X. \& Doolen, G., Diffusion in a multi-component lattice Boltzmann equation model. Technical Report, Theoretical Division, Los Alamos National Laboratory, Los Alamos, NM 87545, 2002.

[15] He, X. \& Li, N., Lattice Boltzmann simulation of electrochemical systems. Computer Physics Communications, 129, pp. 158-166, 2000. doi: http://dx.doi.org/10.1016/ S0010-4655(00)00103-X

[16] Thürey, N., Physically based animation of free surface flows with the lattice Boltzmann method. Doctoral Thesis, Der Technischen Fakultät, Universität Erlangen-Nürnberg, Erlangen-Nürnberg, Germany, 2007.

[17] Pohl, T., High performance simulation of free surface flows using the lattice Boltzmann method, Der Technischen Fakultät, Universität Erlangen-Nürnberg, Erlangen-Nürnberg, Germany, 2008. 